\title{
Electroencephalographic and clinical features of cerebral malaria
}

\author{
J Crawley, S Smith, P Muthinji, K Marsh, F Kirkham
}

\begin{abstract}
Background-Seizures are a prominent feature of childhood cerebral malaria, and are associated with an increased risk of death and neurological sequelae. We present the electroencephalographic (EEG) findings from a detailed clinical and electrophysiological study.

Methods-Children with cerebral malaria had EEGs recorded within six hours of admission, and at $\mathbf{1 2}$ hourly intervals until recovery of consciousness. Ten deeply comatose children underwent intracranial pressure monitoring. Children were not mechanically ventilated, which made it possible to directly correlate the clinical and EEG findings.
\end{abstract}

Results-Of 65 children aged 9 months and above, 40 had one or more seizures, and $18 \mathrm{had}$ an episode of status epilepticus. Most seizures were partial motor, and spike wave activity consistently arose from the posterior temporo-parietal region, a border zone area lying between territories supplied by the carotid and vertebrobasilar circulations. Fifteen children had seizures that were clinically subtle or electrographic. Clinical seizures were associated with an abrupt rise in intracranial pressure. Fifty children recovered fully, seven died, and eight had persistent neurological sequelae. Initial EEG recordings of very slow frequency, or with background asymmetry, burst suppression, or interictal discharges, were associated with an adverse outcome.

Conclusions-Serial EEG recording has uncovered a range of clinical, subtle, and electrographic seizures complicating childhood cerebral malaria, and has emphasised their importance in the pathogenesis of coma. Further work is required to determine the most appropriate regimen for the prophylaxis and treatment of seizures in cerebral malaria, in order to improve outcome.

(Arch Dis Child 2001;84:247-253)

Keywords: cerebral malaria; coma; seizures; electroencephalogram

Health, London, UK

F Kirkham

Correspondence to: Dr J Crawley, Centre for Tropical Medicine, Nuffield Department of Clinical Medicine, Level 7, John Radcliffe Hospital, Headington, Oxford OX3 9DU, UK

jane.crawley@ndm.ox.ac.uk

Accepted 9 October 2000
Prolonged, multiple seizures are a prominent clinical feature, ${ }^{45}$ and are associated with an increased risk of death and neurological
Caused malaria, a diffuse encephalopathy by Plasmodium falciparum, remains a Saharan Africa. ${ }^{1}$ The pathophysiology of the disease is complex and poorly understood. ${ }^{23}$ sequelae..$^{6-8}$ Despite their importance, and in common with many other causes of childhood coma, there have been few descriptions of the clinical and electroencephalographic features of seizures in cerebral malaria. Electroencephalography (EEG) can supply the clinician with information on the origin and distribution of seizure activity, detect subtle or subclinical seizures, and may reveal abnormal electrical activity of prognostic significance. ${ }^{9}$ It is therefore of potential value in the clinical management of cerebral malaria, and may improve understanding of the underlying pathophysiology. Here we present the EEG findings from a detailed clinical and electrophysiological study of 65 children with cerebral malaria. ${ }^{5}$

Methods

STUDY SITE

The study was conducted at the Kenya Medical Research Institute (KEMRI) unit, located at Kilifi District Hospital, on the coast of Kenya. The hospital serves a predominantly rural population, and approximately 5000 children are admitted to the 35 bed paediatric ward annually. Malaria transmission (of which over $95 \%$ is Plasmodium falciparum) occurs throughout the year, with peak transmission following the rainy seasons of April-May and October-November. ${ }^{10}$

PATIENTS

Children aged 9 months and above were eligible for enrolment in the study if they fulfilled the World Health Organisation definition of cerebral malaria, namely unrousable coma not attributable to any other cause in the presence of asexual Plasmodium falciparum parasitaemia. ${ }^{11}$ To fulfil the definition of cerebral malaria, coma had to persist for at least one hour after a seizure and/or after the administration of diazepam. Consent was obtained once all procedures had been fully explained to the child's parent or guardian in their first language.

\section{INVESTIGATIONS}

On admission, baseline blood was taken for parasite count, full blood count, electrolytes, glucose, and venous gas. Blood glucose was subsequently monitored every four hours, and parasite count eight hourly. Lumbar puncture was performed once the level of consciousness was starting to improve. In deeply comatose children, intracranial pressure was monitored using a subarachnoid catheter (Camino 1104B). ${ }^{12}$ 
Table 1 Clinical and laboratory features by outcome

\begin{tabular}{|c|c|c|c|c|}
\hline & $\begin{array}{l}\text { Normal recovery } \\
n=50\end{array}$ & $\begin{array}{l}\text { Sequelae } \\
n=8\end{array}$ & $\begin{array}{l}\text { Died } \\
n=7\end{array}$ & $p$ valuet \\
\hline Age $(\mathrm{mth})^{\star}$ & $33.1(27.6$ to 39.7$)$ & $16.1(9.1$ to 28.5$)$ & $38.7(22.2$ to 67.5$)$ & $\begin{array}{l}0.02 \text { for } 2 v 1 \\
0.04 \text { for } 2 v 3\end{array}$ \\
\hline Duration of coma before admission $(\mathrm{h})^{\star}$ & $6.3(4.7$ to 8.3$)$ & 11.5 (5.0 to 26.4$)$ & 13.3 (6.9 to 25.8$)$ & 0.07 \\
\hline Status epilepticus before or after admission $\ddagger$ & $28 / 50(56 \%)$ & $6 / 8(75 \%)$ & $2 / 7(29 \%)$ & 0.24 \\
\hline Blantyre score 2 or less $\$$ & $35 / 50(76 \%)$ & $5 / 8(63 \%)$ & $7 / 7(100 \%)$ & 0.45 \\
\hline Opisthotonic posturing & $2 / 50(4 \%)$ & $1 / 8(12 \%)$ & $4 / 7(57 \%)$ & 0.003 for $3 v 1$ \\
\hline Median (IQR) parasitaemia (per $\mu \mathrm{l})$ & $85200(990$ to 1017600$)$ & 11854 (1264 to 220000$)$ & $81600(420$ to 352800$)$ & 0.27 \\
\hline Haemoglobin $(\mathrm{g} / \mathrm{l})$ & $65(58$ to 72$)$ & $80(54$ to 106$)$ & $78(50$ to 106$)$ & 0.20 \\
\hline Median (IQR) glucose ( $\mathrm{mmol} / \mathrm{l})$ & $5.9(0.7$ to 11.3$)$ & $5.0(0.7$ to 8.3$)$ & $3.4(0.4$ to 7.8$)$ & 0.17 \\
\hline Median (IQR) sodium (mmol/l) & $134(127$ to 142$)$ & $139(128$ to 168$)$ & $139(127$ to 147$)$ & 0.12 \\
\hline Potassium $(\mathrm{mmol} / \mathrm{l})^{\star}$ & $4.3(4.0$ to 4.5$)$ & $4.7(4.2$ to 5.3$)$ & $4.3(3.2$ to 5.9$)$ & 0.5 \\
\hline Urea $(\mathrm{mmol} / \mathrm{l})^{\star}$ & $4.1(3.3$ to 5.2$)$ & $8.6(3.0$ to 24.7$)$ & $6.5(3.6$ to 11.8$)$ & 0.06 \\
\hline Creatinine $(\mu \mathrm{mol} / \mathrm{l})^{\star}$ & $52(45$ to 61$)$ & $104(55$ to 197$)$ & 72 (38 to 137$)$ & 0.009 for $2 v 1$ \\
\hline Base excess & $-5(-8$ to -3$)$ & $-11(-17$ to -5$)$ & $-13(-21$ to -5$)$ & 0.04 for $3 v 1$ \\
\hline
\end{tabular}

Data are mean (95\% CI) or number (\%) unless stated.

${ }^{\star}$ Geometric mean.

tWhere $\mathrm{p}<0.05$, results of multiple comparison tests (Scheffe or Bonferroni) are given.

$\ddagger$ Any clinical seizure reported (before admission) or observed (after admission) to last for 30 minutes or more.

$\$ 0=$ deep coma, $5=$ full consciousness.

ELECTROPHYSIOLOGY

EEG recordings were made on a 14 channel Medelec 1A94 EEG machine. Silver/silver chloride electrodes were fixed with Elefix and tape to the child's shaved head, and the International 10-20 system used for electrode placement. Recordings were taken within six hours of admission, and at 12 hourly intervals until recovery of consciousness. Continuous recordings using a cerebral function analysing monitor (CFAM, Medaid Ltd, UK) were obtained from selected children who had been unconscious for more than 24 hours or who had prolonged or subtle seizure activity. All EEGs were subsequently analysed by SS, who knew the age of each child and what drugs they had been given, but was blind to any other clinical information.

\section{CLINICAL MANAGEMENT}

Children received standard treatment for cerebral malaria, as detailed previously. ${ }^{5}$ Oxygen was available if required, but there were no facilities for mechanical ventilation, a situation common to most hospitals throughout Africa. All clinical seizures were timed and recorded by medical or nursing staff, and classified as generalised tonic-clonic, partial motor, or partial with secondary generalisation. ${ }^{13}$ Seizures lasting for more than five minutes were treated with a maximum of three doses of diazepam $0.3 \mathrm{mg} / \mathrm{kg}$, given as a slow intravenous injection over two minutes. Children with recurrent (more than three) seizures or status epilepticus (continuous clinical seizure activity lasting for 30 minutes or more) were treated with a loading dose of intravenous phenytoin $18 \mathrm{mg} / \mathrm{kg}$ and, if that failed, with intramuscular phenobarbitone $18 \mathrm{mg} / \mathrm{kg}$. One child with intractable generalised status epilepticus required treatment with intravenous thiopentone $4 \mathrm{mg} / \mathrm{kg}$.

FOLLOW UP

All survivors were seen one month after discharge for neurological examination and repeat EEG. Cerebral computerised tomography (CT) was performed on children with neurological sequelae.
STATISTICS

Analyses were carried out using Stata version 5.0. Student's $t$ test and analysis of variance, with the Scheffe or Bonferroni correction for multiple comparisons, were used for normally distributed quantitative data. Data were log tranformed if the distribution was skewed. The Mann-Whitney test was used for data failing to conform to a normal distribution, and Fisher's exact test for categorical data. Multivariate logistic regression analysis was used to examine the relation between specific admission characteristics and subsequent outcome.

\section{Results}

ADMISSION CHARACTERISTICS

Sixty five children (age range 9 months to 11 years, median 30 months), were recruited to the study. All children had previous normal development. Prior to hospital admission, 68\% (44/65) had seizures which, in 27 cases, were reported to have lasted for at least 30 minutes. In more than half the cases, seizure activity heralded the onset of coma. Children had been comatose for between one and 72 hours (median seven hours) prior to admission and, at the time of admission, $75 \%$ were deeply unconscious with a Blantyre coma score of 2 or less. ${ }^{4}$ Table 1 details clinical and laboratory features of the children.

\section{CLINICAL COURSE}

Fourteen children $(22 \%)$ recovered consciousness within six hours of multiple or prolonged seizures that had occurred on or immediately prior to hospital admission. Following admission, $62 \%(40 / 65)$ had at least one seizure, while 19 children had more than five seizures. Eighteen children (28\%) had an episode of clinical status epilepticus (continuous clinical seizure activity lasting for 30 minutes or more), while three further children had continuous electrical discharges lasting for 30 minutes or more with no apparent clinical correlates (electrographic status epilepticus). Fifty two per cent of the seizures were partial motor, $14 \%$ partial with secondary generalisation, and 34\% generalised tonic-clonic. Children with generalised seizures were significantly older than those with partial motor seizures (mean age 


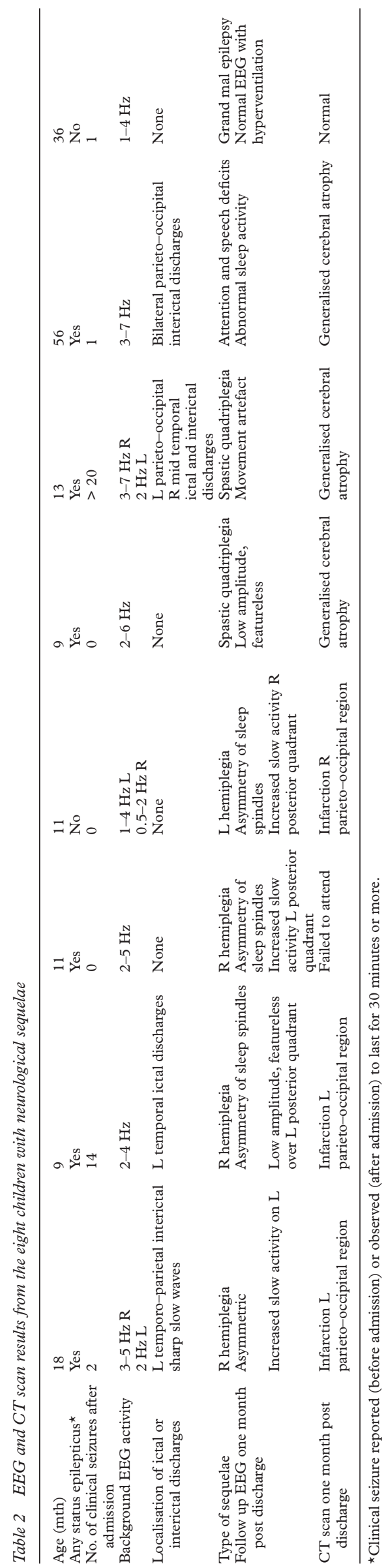

64.0 months (95\% confidence interval (CI) 44.7 to 83.5 ) versus 26.7 months (95\% CI 20.6 to 32.9$) ; \mathrm{p}<0.001)$. Median duration of seizure activity was three minutes (range 0.5-600 minutes). Fifteen children (23\%) had seizures that were either clinically subtle or electrographic.

Median time to recovery of consciousness was 20 hours (range 4-112 hours). Seventy seven per cent $(50 / 65)$ of the children made a full recovery, $11 \%(7 / 65)$ died, and 12\% (8/65) were left with neurological sequelae (hemiplegia in four, spastic quadriplegia in two, cognitive and speech problems in one, grand mal epilepsy in one) that were still present one month after discharge (see table 2).

\section{ELECTROPHYSIOLOGY}

Background activity

Admission EEGs were dominated by high amplitude ( $>100 \mu \mathrm{v})$ slow wave activity with frequencies of $0.5-7 \mathrm{~Hz}$. Admission recordings of very slow frequency $(0.5-3 \mathrm{~Hz})$ were obtained from $17 / 65(26 \%)$ children, and were associated with an increased risk of death (odds ratio 25.6 (95\% CI 2.8 to 235$) ; \mathrm{p}=0.004$; table 3 ). This association remained significant despite adjustment for the possible confounding effects of age, glucose, and base excess. Recordings of very slow frequency were not significantly associated with duration of coma, administration of diazepam in the 12 hours prior to admission EEG, age, parasitaemia, glucose, or base excess $(\mathrm{p}>0.1$ for all comparisons). Ten children had admission recordings that were asymmetric, with increased slow wave activity over all or part of one hemisphere. Nine of these children had a history of multiple partial motor seizures or status epilepticus in the six hour period prior to admission, and three developed neurological sequelae.

Ictal activity

A striking feature of the EEGs in this series was the consistent localisation of ictal discharges over the posterior temporo-parietal regions. Over $75 \%$ of ictal or interictal discharges occurred in this region, sometimes spreading to involve both temporo-parietal regions, or one or both cerebral hemispheres. In eight cases, electrical seizure activity persisted for between two and 140 minutes (median 45 minutes) after successful treatment of a clinical seizure with diazepam. Fifteen children had continuous electrical seizure discharges on EEG, but clinical features that were either extremely subtle or not discernible (electrographic). These children typically presented in coma, with a history of a prolonged partial seizure that had either terminated spontaneously, or as a result of treatment with diazepam. Tonic eye deviation, nystagmus, salivation, and hypoventilation were the most distinctive clinical features. ${ }^{14}$ The nystagmus was of large amplitude, with a slow phase of movement that crossed the midline. Respiration was shallow and irregular, and the children were hypoxaemic (arterial oxygen saturation below $80 \%$ ) and hypercarbic $\left(\mathrm{pCO}_{2}\right.$ above $\left.6.5 \mathrm{kPa}\right)$. EEG showed continuous 


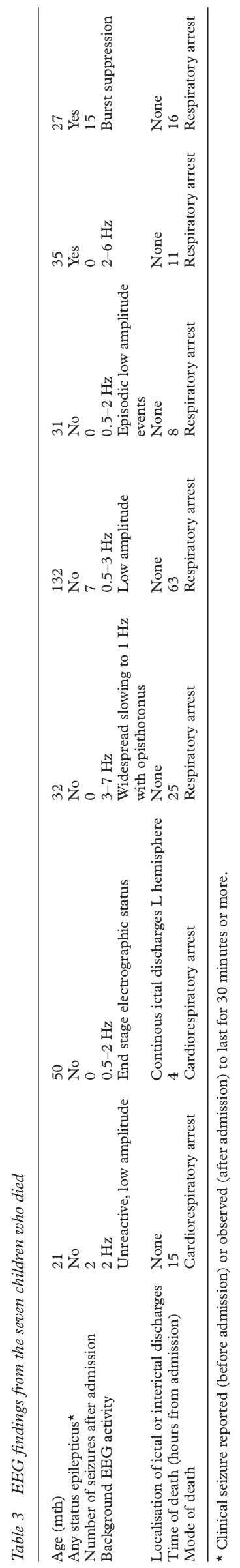

spike wave discharges over the posterior temporo-parietal region contralateral to the direction of eye deviation.

The onset of generalised electrical and clinical seizure activity usually followed an increased frequency of bilateral, predominantly posterior, interictal spike wave discharges. Runs of up to 40 short, generalised seizures, each lasting for 0.5 to 1 minute, were observed in four children, and were followed by notable postictal flattening. Clinical features became increasingly subtle during prolonged generalised seizures. One child was admitted following a generalised seizure at home that had lasted for six hours. Tonic-clonic movements had ceased one hour before admission, and the child was deeply comatose, with irregular respiration, salivation, and priapism. Despite the paucity of clinical features, EEG revealed continuous, generalised seizure activity.

Interictal epileptic activity

Interictal spikes and sharp slow waves were observed on admission EEGs from three children, all with a history of prolonged (seizure activity lasting for more than one hour) partial motor status epilepticus. The discharges were bilateral and multifocal, and located predominantly in the temporo-parietal regions (table 2). All of these children developed neurological sequelae.

Episodic low amplitude events

Periods of relative attenuation of background activity lasting three to five seconds were seen on admission EEGs from five children. In two cases, these followed administration of intravenous diazepam $0.3 \mathrm{mg} / \mathrm{kg}$ for the treatment of seizures. Four of these children recovered normally, while one child died seven hours after admission.

Burst suppression

A burst suppression pattern, consisting of bursts of polymorphous complexes occurring synchronously over both hemispheres, and alternating with periods of relative quiescence, was observed on the initial EEGs from two children. One child had been hypotensive and hypoglycaemic on admission, and the other had a history of multiple, prolonged generalised seizures. Both children died.

Intracranial pressure monitoring and EEG

Ten children had simultaneous EEG and intracranial pressure (ICP) monitoring. Four children had clinical seizures during ICP monitoring. Intracranial pressure rose by a median of $164 \%$ (range 108-285\%) during generalised seizures, and by $50 \%$ (range 0-186\%) during partial seizures. Concomitant rise in mean arterial pressure meant that cerebral perfusion pressure was maintained above $50 \mathrm{~mm} \mathrm{Hg}$ during clinical seizures. There was no significant rise in ICP during two 90 minute periods of electrographic seizure activity confined to the posterior temporoparietal regions. At intracranial pressures of below $20 \mathrm{~mm} \mathrm{Hg}$, fluctuations in ICP appeared to have no effect on the background frequency 
A

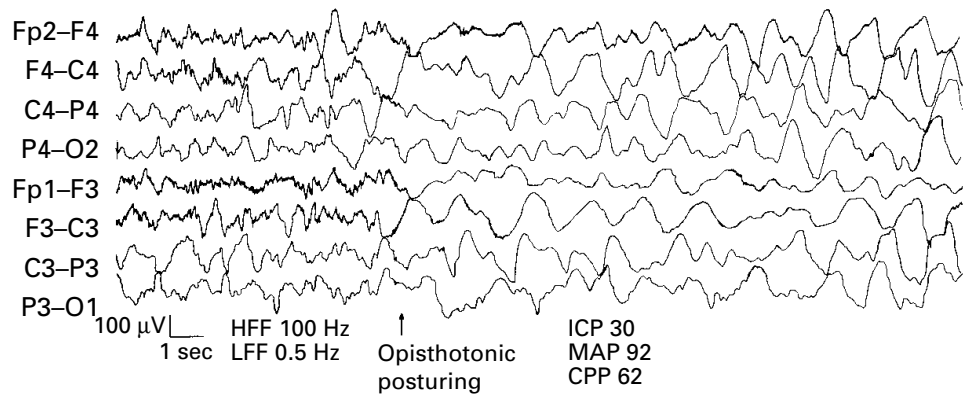

B

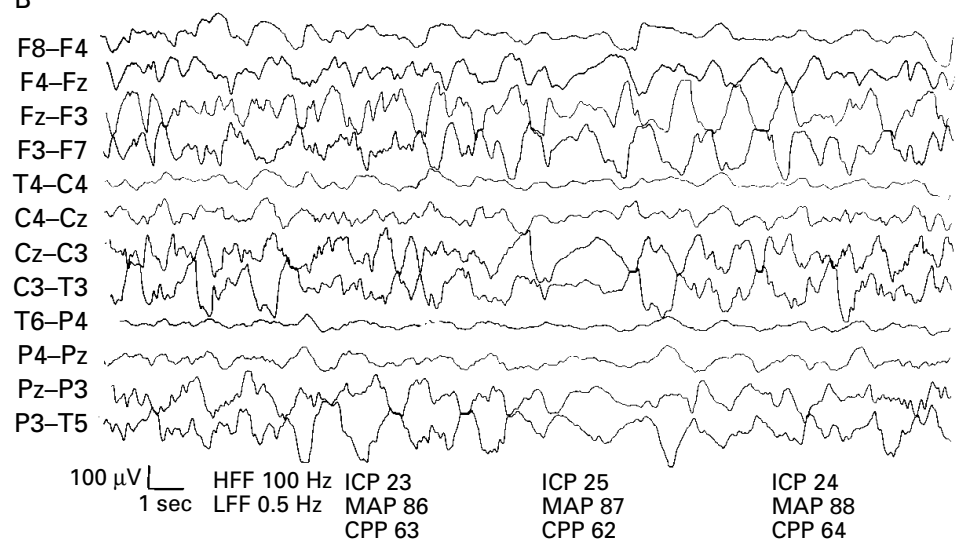

Figure 1 (A) EEG recording showing widespread slow wave activity in association with opisthotonic posturing (onset indicated with an arrow). (B) EEG recorded 10 hours later. There is notable attenuation of electrical activity in the right parieto-temporal region (leads T4-C4, C4-Cz, T6-P4, P4-Pz). ICP (intracranial pressure), MAP (mean arterial pressure), and CPP (cerebral perfusion pressure) measured in $\mathrm{mm} \mathrm{Hg}$.

of the EEG. EEG recording from one child with severe intracranial hypertension, however, showed widespread slow wave activity (frequencies of $0.5-1 \mathrm{~Hz}$ ) in association with episodes of opisthotonic posturing, during which ICP rose to peaks of 30 to $40 \mathrm{~mm} \mathrm{Hg}$ (fig 1A). Within 10 hours, EEG showed attenuation of activity in the right temporoparietal region (fig 1B), and the right pupil became dilated with sluggish reaction to light. Over the next five hours, despite regular doses of mannitol $0.5 \mathrm{~g} / \mathrm{kg}$, the ICP rose to above 80 $\mathrm{mm} \mathrm{Hg}$, the cerebral perfusion pressure fell below $30 \mathrm{~mm} \mathrm{Hg}$, and the entire EEG became flat and featureless. ICP rose to a peak of 125 $\mathrm{mm} \mathrm{Hg}$, and the child died following a respiratory arrest.

Another child aged 3 years was admitted with a 12 hour history of opisthotonic posturing. Initial EEG was reactive, with a background frequency of 2-6 Hz. ICP monitoring was commenced at seven hours, and the child also started continuous CFAM recording. Initial intracranial pressures ranged from 25 to 30 $\mathrm{mm} \mathrm{Hg}$, with peaks of $40 \mathrm{~mm} \mathrm{Hg}$ during episodes of opisthotonus. Over the next four hours, despite inotropic support, mean arterial pressure remained below $75 \mathrm{~mm} \mathrm{Hg}$, and rising intracranial pressure consequently caused a progressive decline in cerebral perfusion pressure. Subsequent infusion of mannitol was accompanied by a notable deterioration in clinical condition, and CFAM recording showed a sharp drop in background frequency.
The child died shortly afterwards following a cardiorespiratory arrest.

\section{OUTCOME}

Serial EEGs showed a gradual increase in background frequency over the period from admission to recovery of consciousness. The 50 children who made a full recovery had normal EEGs one month after discharge. Children with neurological sequelae were significantly younger $(p=0.02)$ and had a significantly higher creatinine $(p=0.009)$ compared to those who recovered fully. Admission base excess was significantly greater $(p=0.04)$ in children who died compared to those who recovered fully, while four of the seven children who died had sustained opisthotonic posturing during their clinical course in hospital. There were otherwise no significant differences between the three outcome groups in the admission variables, but numbers are small. Follow up EEGs from children with hemiplegia showed decreased activity in the contralateral parieto-temporal region, and cerebral CT scans showed infarction in the same region. Follow up EEGs from two children with spastic quadriplegia and one child with severe cognitive and speech problems were of low amplitude, with a paucity of normal cerebral rhythms, while CT scans from these children showed global cerebral atrophy (table 2).

\section{Discussion}

Traditionally, it has been assumed that sequestration of parasitised red blood cells occurs within the cerebral microvasculature of all comatose patients with falciparum malaria. ${ }^{11} 15$ Clinical and experimental work has shown, however, that the pathophysiology of cerebral malaria is highly complex, and that a number of different pathological mechanisms may cause coma. ${ }^{2}$ Reduced cerebral perfusion (secondary to raised intracranial pressure and systemic hypotension), severe anaemia, hypoglycaemia, and the local release of cytokines and nitric oxide may all be contributory factors. ${ }^{3} 121617$ Our study suggests that an additional pathophysiological factor, seizures, may play an important role in the pathogenesis of coma.

Over one third of the children in this study had more than five seizures or an episode of status epilepticus during their clinical course in hospital. Twenty two per cent recovered consciousness within six hours of prolonged or multiple seizures, suggesting that their coma may have been a postictal phenomenon, directly related to seizures. EEG was essential for identifying the 15 children in whom seizures were either clinically subtle (tonic eye deviation, nystagmus, hypoventilation) or electrographic. Although tonic deviation of the head and eyes commonly occurs during epileptic seizures, nystagmoid eye movements are an unusual ictal manifestation. The clinical characteristics of the nystagmus in these patients were consistent with epileptic nystagmus type two (EN-2), which typically has a seizure focus in the temporo-parietal-occipital cortex, contralateral to the direction of the nystagmus beats. ${ }^{18} 19$ This area overlaps the 
putative cortical region of visual motor sensitivity (V5), which is important for the generation of ipsiversive smooth pursuit. ${ }^{20}$ Subtle seizures have not been described in previous studies of childhood cerebral malaria but are of great clinical importance, as several of these children recovered consciousness within a few hours of anticonvulsant treatment. In a busy, understaffed hospital in the developing world such seizures are easily missed, yet their clinical features are sufficiently distinct for them to be recognised by medical or nursing staff with appropriate training, without the need for EEG.

The pathogenesis of seizures in childhood cerebral malaria is likely to be complex and multifactorial, ${ }^{5}$ but our study suggests that cerebral hypoxia may be a precipitating factor. EEG recordings showed that electrical seizure activity consistently arose from the posterior temporo-parietal region, which is a "watershed" area, lying between territories supplied by the middle cerebral (carotid circulation) and posterior cerebral (vertebro-basilar circulation) arteries. Consequently, it is particularly vulnerable to hypoxia when oxygen delivery to the brain is compromised as a result of sequestration, severe anaemia, or inadequate cerebral perfusion caused by hypotension or raised intracranial pressure. ${ }^{12} 152122$ Because of their anatomical position close to the temporal lobes in the tentorial notch, the posterior cerebral arteries may be particularly vulnerable to compromise in those patients who have acute intracranial hypertension and incipient transtentorial herniation, as illustrated by the EEGs in fig 1. Raised intracranial pressure, reduced cerebral perfusion pressure, and transtentorial herniation have been documented in cerebral malaria, ${ }^{12} 23$ and in other paediatric encephalopathies. ${ }^{24}$ By initiating the release of excitotoxic mediators such as glutamate or quinolinic acid, ${ }^{25}$ local hypoxia may precipitate seizures, which, by raising intracranial pressure and increasing the demand for oxygen and glucose, may exacerbate the situation further. Although there is no evidence for failure of cerebral autoregulation in the majority of these patients, relatively poor collateral circulation in some patients may mean that cerebral blood flow in the border zone between the carotid and vertebro-basilar territories is insufficient to compensate for increased local demand. In these patients, a vicious cycle might then be generated, leading to intractable partial seizures and eventually to focal infarction (table 2).

Diffuse background slow wave activity on EEG occurs in many conditions (metabolic, toxic, and infectious) that have a generalised effect on the brain. ${ }^{92}$ Background slow wave activity in cerebral malaria has been described in adults, ${ }^{27-29}$ and in one previous study on children. ${ }^{30}$ The mechanism for the increased slow wave activity seen during episodes of intracranial hypertension and opisthotonus is unknown, but may have been caused by concurrent hyperventilation, leading to hypocapnia and cerebral vasoconstriction. ${ }^{31}$ Intracranial pressure rose acutely during seizures, and was accompanied by a concurrent rise in mean arterial pressure, suggesting that cerebral autoregulation was intact. In one case, however, mean arterial pressure was insufficient to maintain adequate cerebral perfusion, despite treatment with pressor agents. Infusion of mannitol was followed by clinical and electrophysiological deterioration, suggesting that the integrity of the blood-brain barrier had been breached.

EEGs have been used to predict prognosis in a number of childhood encephalopathies. ${ }^{32-34}$ In this study, burst suppression or background activity of very slow frequency was associated with an increased risk of death. Of five children with episodic low amplitude events on initial EEG, four made a full recovery. In two of these cases, episodic low amplitude events were associated with the administration of diazepam, which may partly explain why their prognosis appears better here than in other types of encephalopathy. ${ }^{35}$ Children with a history of status epilepticus prior to admission, and an initial EEG showing asymmetric background activity or continuous interictal multifocal spike waves, had an increased incidence of neurological sequelae. Overall, almost $70 \%$ of the children with an adverse outcome (death or neurological sequelae) had one or more poor prognostic features on initial EEG. Although this suggests that in some cases significant cerebral pathology must have occurred prior to hospital admission, anticonvulsant prophylaxis given at the time of admission may prevent further cerebral damage. A recent randomised, controlled study of phenobarbitone prophylaxis in childhood cerebral malaria showed that, at a dose of $20 \mathrm{mg} / \mathrm{kg}$, phenobarbitone halved seizure frequency but was associated with an unacceptable doubling of mortality. ${ }^{37}$ Further studies need to be done to find a prophylactic anticonvulsant drug that is both safe and effective in children with cerebral malaria.

Electrophysiological monitoring using serial EEG and CFAM has made it possible to identify a wide variety of seizures (clinical, subtle, and electrographic) in children with cerebral malaria, and has emphasised their importance in the pathogenesis of coma. We have been able to correlate the electroencephalographic and clinical findings from this group of unconscious children, something that is not possible in most parts of the developed world where the majority of unconscious children are ventilated. The information obtained from this study may, therefore, provide some valuable insights into a wide variety of other paediatric encephalopathies.

This paper is published with the permission of the Director of KEMRI. We wish to thank our clinical and nursing colleagues on the paediatric research ward at Kilifi District Hospital for their skilled help with patient management. The work is supported by KEMRI and by the Wellcome Trust (040313). We are also extremely grateful to the British High Commission in Nairobi, and the Triangle Trust for additional support. KM is a Wellcome Trust Senior Research Fellow in Clinical Science (031342), and FK was supported by the Wellcome Trust (035352).

\footnotetext{
1 WHO. Malaria, 1982-1997. Wkly Epidemiol Rec 1999;74:

2 Marsh K, English M, Crawley J, Peshu N. The pathogenesis Marsh K, English M, Crawley J, Peshu N. The pathogenesis
of severe malaria in African children. Ann Trop Med Parasitol 1996;90:395-402.
} 
3 English M, Marsh K. Childhood malaria-pathogenesis and treatment. Curr Opin Infect Dis 1997;10:221-5.

Molyneux ME Taylor TE Wirima JJ Borgstein A Clinical features and prognostic indicators in paediatric cerebral malaria: a study of 131 comatose Malawian children. $Q 7 M$ 1989;71:441-59.

5 Crawley J, Smith S, Kirkham F, et al. Seizures and status epilepticus in childhood cerebral malaria. $Q f M$ 1996;89: 591-7.

6 Jaffar S, Van Hensbroek MB, Palmer A, et al. Predictors of a fatal outcome following childhood cerebral malaria. Am Trop Med Hyg 1997;57:20-4.

7 Brewster DR, Kwiatkowski D, White NJ. Neurological sequelae of cerebral malaria in children. Lancet 1990;336 1039-43.

8 Bondi FS. The incidence and outcome of neurological abnormalities in childhood cerebral malaria: a long-term follow up of 62 survivors. Trans R Soc Trop Med Hyg 1992; 86:17-19.

9 Hughes JR. EEG in clinical practice, 2nd edn. Boston: Butterworth-Heinemann, 1994

10 Snow RW, Armstrong-Schellenberg JRM, Peshu N, et al. Periodicity and space-time clustering of severe childhood malaria on the coast of Kenya. Trans $R$ Soc Trop Med Hyg 1993;87:386-90

11 WHO. Severe falciparum malaria. Trans $R$ Soc Trop Med Hyg 2000;94(suppl 1):1-90

12 Newton CRJC, Crawley J, Sowumni A, et al. Intracranial hypertension in Africans with cerebral malaria. Arch Dis Child 1997;76:219-26.

13 Hopkins A, Shorvon S, Cascino G, eds. Epilepsy, 2nd ed. London: Chapman and Hall Medical, 1995.

14 Crawley J, English M, Waruiru C, et al. Abnormal respiratory patterns in childhood cerebral malaria. Trans $R$ Soc Trop Med Hyg 1998;92:305-8.

15 MacPherson GG, Warrell MJ, White NJ, et al. Human cerebral malaria. A quantitative ultrastructural analysis of parasitized erythrocyte sequestration. Am f Pathol 1985; parasitized eryth

16 White NJ, Miller KD, Marsh K, et al. Hypoglycaemia in African children with severe malaria. Lancet 1987;1:70811.

17 Clark I, Rockett K, Cowden W. Proposed link between cytokines, nitric oxide, and human cerebral malaria. Parasitology Today 1991;7:205-7.

18 Kaplan PW, Tusa RJ. Neurophysiologic and clinical correlations of epileptic nystagmus. Neurology 1993;43:2508-14.

9 Harris CM, Boyd S, Chong K, et al. Epileptic nystagmus in infancy. $\mathcal{F}$ Neurol Sci 1997;151:111-14.

20 Lekwuwa GU, Barnes GR. Cerebral control of eye movements. I. The relationship between cerebral lesion sites and smooth pursuit deficits. Brain 1996;119(pt 2):473-90.
21 English M, Muambi B, Mithwani S, Marsh K. Lactic acidosis and oxygen debt in African children with severe sis and oxygen debt in Africa

22 Newton CRJC, Peshu N, Kendall B, et al. Brain swelling and ischaemia in Kenyans with cerebral malaria. Arch Dis Child 1994;70:281-7.

23 Newton CRJC, Kirkham FJ, Winstanley PA, et al. Intracranial pressure in African children with cerebral malaria. Lancet 1991;337:573-6.

24 Minns RA. Problems of intracranial pressure in childhood. London, Oxford: Blackwell Scientific Publications Ltd, 1991.

25 Dobbie M, Crawley J, Waruiru C, et al. Cerebrospinal fluid studies in children with cerebral malaria: an excitotoxic mechanism? Am f Trop Med Hyg 2000;62:284-90.

26 Plum F, Posner JB. The diagnosis of stupor and coma, $3 \mathrm{rd}$ edn. Philadelphia: FA Davis Company, 1982.

27 Stotka VL, Wenzel RP. Malaria in Vietnam (1 Corps Sector): review of 214 cases including EEG patterns on 19 acutely ill patients. Mil Med 1973;138:795-802.

28 Chen SS, Way LJ, Chen MC, Chen ER. Clinical and electrophysiological assessment of cerebral malaria. Kaohelectrophysiological assessment
siung f Med Sci 1991;7:278-84.

29 Thumasupapong S, Tin T, Sukontason K, et al. Electroencephalography in cerebral malaria. SE Asian $\mathcal{F}$ Trop Med Public Health 1995;26:34-7.

30 Lemercier G, Bert J, Nouhouayi A, et al. Le neuropaludisme: aspects electroencephalographiques, neuropathologiques, problemes physiopathologiques. Pathologie-Biologie 1969;17:459-72.

31 Kiloh LG, McComas AJ, Osselton JW, Upton ARM. Clinical electroencephalography, 4th edn. London: Butterworths, 1981.

32 Pampiglione G, Harden A. Resuscitation after cardiocirculatory arrest: prognostic evaluation of early electroencephalographic findings. Lancet 1968;1:1261-5.

33 Kuroiwa Y, Celesia GG. Clinical significance of periodic EEG patterns. Arch Neurol 1980;37:15-20

34 Tasker RC, Boyd S, Harden A, Matthew DJ. Monitoring in non-traumatic coma. Part II: electroencephalography. Arch Dis Child 1988;63:895-9.

35 Brenner RP, Schwartzman RJ, Richey ET. Prognostic significance of episodic low amplitude or relatively isoelectric EEG patterns. Dis Nerv Syst 1975;10:582-7.

36 Rae-Grant AD, Strapple C, Barbour PJ. Episodic lowamplitude events: an under-recognised phenomenon in clinical electroencephalography. F Clin Neurophysiol 1991; 8:203-11

37 Crawley J, Waruiru C, Mithwani S, et al. Effect of phenobarbital on seizure frequency and mortality in childhood cerebral malaria: a randomised, controlled intervention study. Lancet 2000;355:701-6. 